\title{
Time-resolved FUSE photometric and spectroscopic observations: PG 1219+534, PG 1605+072, and PG 1613+426
}

\author{
Kuassivi $^{1}$, A. Bonanno ${ }^{2}$, and R. Ferlet ${ }^{3}$
}

1 AZimov association 14, rue Roger Moutte, 83270 St Cyr, France

e-mail: martial.andre@free.fr

2 INAF - Osservatorio Astrofisico di Catania, Citta Universitaria, 5123 Catania, Italy e-mail: abo@ct.astro.it

3 Institut d'Astrophysique de Paris, 98 bis Bd Arago, 75014 Paris, France

e-mail: ferlet@iap.fr

Received 27 February 2005 / Accepted 22 June 2005

\section{ABSTRACT}

We report the detection of pulsations in the far ultraviolet (FUV) light curves of PG 1219+534, PG 1605+072 and PG $1613+426$ obtained with the Far Ultraviolet Spectroscopic Explorer (FUSE) in time-tagged mode (TTAG). Exposures of the order of a few ksec were sufficient to observe the main frequencies of PG 1219+534 and PG 1605+072 and confirm the detection of a pulsation mode at the surface of PG 1613+426 as reported from ground. For the first time we derive time-resolved spectroscopic FUSE data of a sdB pulsator (PG 1605+072) and comment on its line profile variation diagram (lpv diagram). We observe the phase shift between the maximum luminosity and the maximum radius to be consistent with the model of an adiabatic pulsator. We also present evidence that the line broadening previously reported is not caused by rotation but is rather an observational bias due to the rapid Doppler shift of the lines with $17 \mathrm{~km} \mathrm{~s}^{-1}$ amplitude. Thus our observations do not support the previous claim that PG $1605+072$ is (or will evolve into) an unusually fast rotating degenerate dwarf. These results demonstrate the asteroseismological potential of the FUSE satellite which should be viewed as another powerful means of investigating stellar pulsations, along with the MOST and COROT missions.

Key words. instumentation: photometers - instumentation: spectrographs - line: profiles - stars: white dwarfs techniques: radial velocities - stars: oscillations (including pulsations)

\section{Introduction}

Subluminous $\mathrm{B}(\mathrm{sdB})$ stars dominate the population of faint blue stars of our own Galaxy and are numerous enough to account for the UV upturn phenomenon (Brown et al. 2000) observed in elliptical galaxies and galaxy bulges. Since the discovery of sdB stars in the globular cluster NGC 67524 (Heber et al. 1986), evidence has accumulated that sdB stars represent a late stage of stellar evolution. These are evolved objects with typical helium $(\mathrm{He})$ burning cores of $0.5 M_{\odot}$ surrounded by a thin $\mathrm{H}$ surface layer (less than $2 \%$ of the mass) and are located near the extreme horizontal branch (EHB) with effective surface temperatures ranging from $20000 \mathrm{~K}$ to $40000 \mathrm{~K}$. Although important questions remain regarding the exact evolutionary path and time-scales, sdB stars are widely believed to be immediate progenitors of low mass white dwarfs. Most of these subdwarfs reside in close binaries which suggests a formation scenario involving binary interaction and leads to the general expectation that these associations are progenitors of type Ia supernovae.

Since 1997 , the discovery of multi-mode, short period $(P=$ 2-10 min) oscillations among sdBs (Kilkenny et al. 1997) has provided an unique opportunity for probing their interiors using asteroseismological methods. From a theoretical viewpoint (Charpinet et al. 1996), the sdB instability strip has long been predicted to occur between $29000 \mathrm{~K}$ and $37000 \mathrm{~K}$ which seems in good agreement with recent observations. However, relatively few sdB stars in this temperature range are reported to show luminosity variations and it is not clear whether this is a bias due to the poor detection limit from the ground or the effect of some intrinsic physical process. The limitation set by the atmospheric scintillation makes mandatory the use of space-based observatories for further asteroseismological investigations.

Many high-resolution FUV spectra of sdB stars have been made available thanks to the FUSE satellite (Fontaine \& Chayer 2004), but it is only recently that detections of pulsation modes with the FUSE detector have been reported by Kuassivi et al. (2004) towards PG 1219+534. Several previous successful detections of stellar pulsation from space with the Hubble Space Telescope (HST) have been reported in the literature (Kepler et al. 2000) with similar performances. However, until now the lack of high-speed time-resolved spectroscopic data prevented any direct evidence for mode identification from being collected. 
Table 1. Observation summary.

\begin{tabular}{|c|c|c|c|c|c|c|}
\hline Stars & $\begin{array}{r}\text { RA } \\
\text { J2000 }\end{array}$ & $\begin{array}{r}\text { Dec } \\
\text { J2000 }\end{array}$ & $\begin{array}{l}\text { Mag } \\
V\end{array}$ & $\begin{array}{l}\text { Date of } \\
\text { observation* }\end{array}$ & $\begin{array}{l}\text { Exposure } \\
\text { time (s) }\end{array}$ & $\begin{array}{l}\text { Program } \\
\text { ID }\end{array}$ \\
\hline PG $1219+534$ & 122129 & +530437 & 12.4 & 2001-01-16 & 6300 & B033 \\
\hline PG $1605+072$ & 160804 & +070429 & 12.8 & 2001-07-27 & 4700 & B033 \\
\hline PG $1613+426$ & 161447 & +422736 & 14.4 & $2002-07-15$ & 12070 & Z904 \\
\hline
\end{tabular}

* All the targets have been observed through the large FUSE aperture (LWRS).

The advent of the COROT space mission (Baglin et al. 2001) will improve by many orders of magnitude the sensitivity of photometric investigations and bring important clues to the general field of stellar pulsations. However, even the mostawaited COROT space mission, which is designed as a high precision, wide field, stellar photometric instrument, will not provide time-resolved spectroscopic data and will only focus on stellar pulsators brighter than the 9th mag, thus excluding many faint oscillators and most (if not all) sdB stars from further investigation.

In this paper we make use of the unexploited FUSE photometric possibilities and provide an analysis of FUV photometric data collected towards three sdB stars. The observation and the special reduction technique are described in Sects. 2 and 3. The frequency analyzes are summarized in Sect. 4 for each target. In Sect. 5, we focus on the bright PG 1605+072 and derive the first line profile variation (lpv) diagram ever obtained for a sdB star. Future prospects for space-based asteroseismology with the FUSE satellite are discussed in Sect. 6 and a summary is given in Sect. 7 .

\section{Observation}

The FUSE design consists of four co-aligned optical channels (LiF1-2 and SiC1-2); each channel is made up of a telescope primary mirror (35 $\mathrm{cm}$ aperture), a Focal Plane Assembly (FPA) containing the spectrograph entrance apertures, a holographic diffraction grating and a portion of a detector. The FUSE mission, its planning and its on-orbit performance are discussed by Moos et al. (2000) and Sahnow et al. (2000).

A summary of the FUSE observation used in this work is shown in Table 1. The integrated spectra computed by the FUSE pipeline (CalFUSE 2.4) allow for a spectral resolution of 20000 and a signal-to-noise per resolution element $(0.06 \AA$ ) varying from 5 for PG $1219+534$ to 10 for PG 1605+072.

\section{Data reduction}

The reduction of the raw two-dimensional FUSE detector images into useful FUV photometric series has been described in Kuassivi et al. (2004). It proceeds in the three following steps.

First, the photometric time-series is flagged for known instrumental defects correlated with the satellite orbital motion such as pointing drifts and burst events. Pointing drifts are caused by thermal fluctuations within the satellite that perturb the fine co-alignment of the four optical channels. This phenomenon infrequently results in the loss of one or two channels but rarely affects observations made through the large aperture (LWRS). Intermittent increases in the count rate, known as burst events, are a more important issue for photometric calibration and need to be cautiously screened. These events are due to scattered light entering the telescope and occur preferentially in orbital morning. Since the burst events fully illuminate the detector, a few off spectrum regions are carefully monitored so as to characterize these burst events in amplitude and duration. The same regions serve also to monitor the background arrays that are found to be essentially constant throughout the exposures.

The second step consists of flagging regions of the raw image affected by detector oddities such as dead-pixels or moving shadows. At variance with dead pixels whose positions are cataloged and whose impact on the total observed flux is negligible, the moving shadows, known as "worm effects", can reduce the flux by as much as $50 \%$ over a narrow spectral range (typically between 110 and $120 \mathrm{~nm}$ ). Such structures are observed in the $\mathrm{LiF} 1 \mathrm{~A}, \mathrm{LiF} 1 \mathrm{~B}$ and $\mathrm{LiF} 2 \mathrm{~A}$ channels for all our observations and result in a net loss of about $10 \%$ of the total flux.

The last step consists of excluding the airglow emission features whose orbital modulation affect the signal. The photometric time-series are then computed from the photon count rate within these cleaned regions of the detector.

The same procedure is performed for each detector and each exposure. Although most of the reduction needs to be done by hand, many user-friendly IDL applets are available to handle FUV raw data at the FUSE web site. In particular, the FUSE_SCAN procedure allows one to go through each of the above-mentioned steps.

\section{Frequency analysis}

\section{1. $P G 1219+534$}

FUSE time-tagged data have been obtained for the known short period pulsating sdB type star PG 1219+534 (Koen et al. 1999). The data were acquired on the 16th January 2001 for $6300 \mathrm{sec}-$ onds and were reduced following the special procedure outlined by Kuassivi et al. (2004) (see Sect. 3). Figure 1 shows the Direct Fourier Transform (DFT) periodogram computed for a continuous exposure of $3200 \mathrm{~s}$ and the confidence level for the main frequency. A simultaneous non-linear least square fit of two sinusoids provide the two main frequencies: $6.9 \mathrm{mHz}$ and $7.8 \mathrm{mHz}$. Our result is consistent, within our frequency resolution $(0.2 \mathrm{mHz})$, with the result from ground observations (Koen et al. 1999). Hence, the two peaks observed in the FUSE 


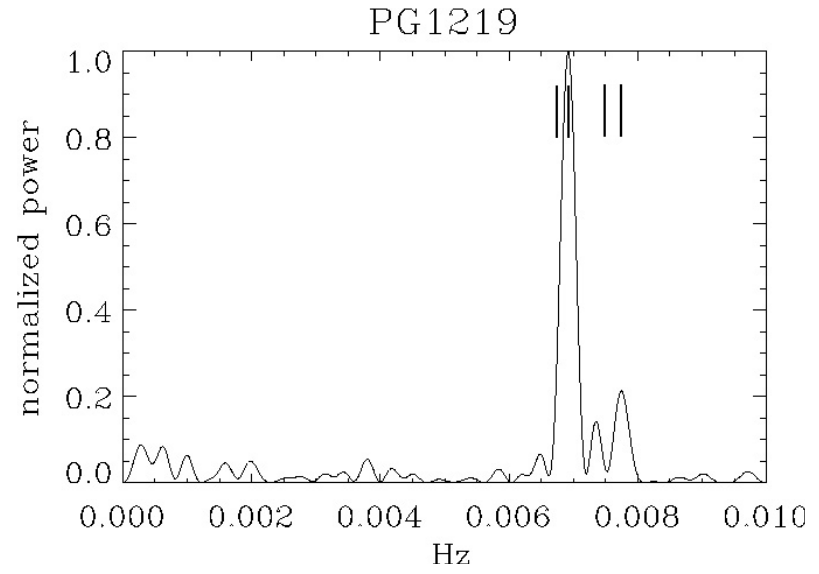

Fig. 1. DFT periodogram obtained towards PG $1219+534$. The vertical lines show the ground based frequencies (Koen et al. 1999).

periodogram (Fig. 1) are indeed a pair of unresolved components: $6.7-7.0 \mathrm{mHz}$ and $7.5-7.8 \mathrm{mHz}$. We find that the relative importance of the two observed frequencies, as far as the power concentration is concerned, is inconsistent with the amplitude spectra obtained by Koen et al. (1999) three years before. At that time, the authors reported small variations in mode amplitude occuring over a few weeks. Our observations suggest larger variations over longer time-scales resembling those reported towards PG $1605+072$ by O'Toole et al. (2002).

\section{2. $P G 1605+072$}

This star seems to be peculiar among sdB stellar pulsators (Kilkenny et al. 1999): it shows the richest power spectrum (up to 50 modes), the longest period (550 s) and the lowest surface gravity $(\log g \approx 5.25)$. It has been proposed that this star has just left the EHB and is now slowly turning into a low mass WD. We obtain the two main frequencies of $2.1 \mathrm{mHz}$ and $2.7 \mathrm{mHz}$ from non-linear sinusoidal fit (see Fig. 2). These large amplitude oscillations are clearly visible in the derived FUV light curve (see Sect. 5.3) and have the same amplitude as the ones observed in the optical domain $(0.25 \mathrm{mag})$ as expected for low- $g$ dwarf stars (Kepler et al. 2000).

\section{3. $P G 1613+426$}

This star is believed to lie near the hot end of the sdB instability trip and is thus expected to show pulsations. Photometric observations of PG $1613+426$ were carried out with the $91-\mathrm{cm}$ Cassegrain telescope of the M. G. Fracastoro stellar station of the Catania Astrophysical Observatory in between June and August 2002 collecting more than $45000 \mathrm{~s}$ of exposure. These observations allowed the detection of a dominant frequency at $6.936 \pm 0.003 \mathrm{mHz}$ while no additional periods could be detected (Bonanno et al. 2003).

In a single $12070 \mathrm{~s}$ FUSE observation, a non-linear least square fit of a multi-periodic sinusoid allows the detection the main frequency peak at $7.048 \pm 0.3 \mathrm{mHz}$ with a confidence level above $90 \%$ (see Fig. 3). Unfortunately severe aliasing prevented us to identify other possible pulsation modes but this

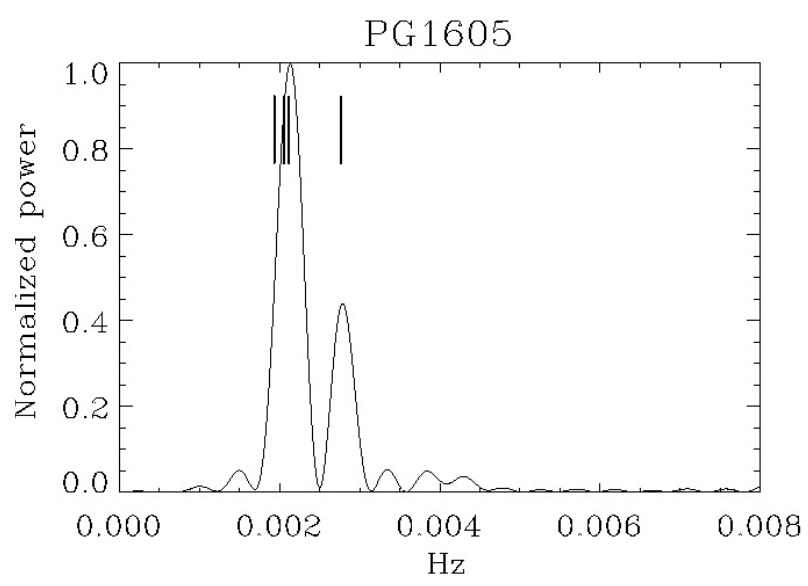

Fig. 2. DFT periodogram obtained towards PG 1605+072. The vertical lines show the four dominant ground based frequencies in this frequency domain (O'Toole et al. 2002).

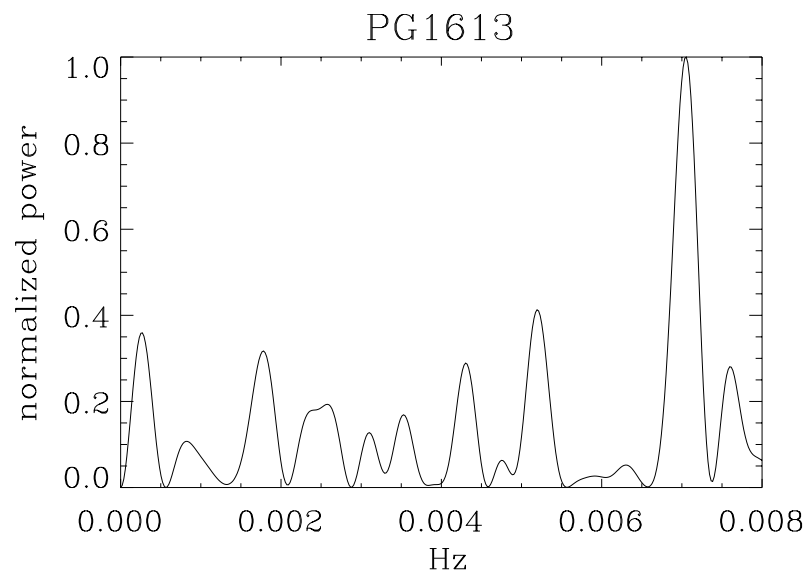

Fig. 3. DFT periodogram obtained towards PG 1613+426. The dominant peak coincides with the previous ground based detection at $6.9 \mathrm{mHz}$ (Bonanno et al. 2003).

limitation could be avoided in the future with a different planning of the FUSE observations.

\section{Time resolved FUV spectroscopy of PG 1605+072}

\subsection{Previous observations}

Several attempts have been made to monitor the spectral variations of sdB pulsators, in particular PG 1605+072, as well as to model them. Although PG $1605+072$ is considered the best target from the observational point of view it is also famous for being a challenging pulsator in terms of interpretation.

Soon after its discovery (Kilkenny et al. 1999), PG 1605+072 was selected for a detailed quantitative spectral analysis. The richness of its pulsation spectrum was simultaneously interpreted by Kawaler et al. (1999) as an effect of a $g$-trapped mode in an extremely fast rotating star: $v_{\mathrm{rot}} \approx 130 \mathrm{~km} \mathrm{~s}^{-1}$. This prediction found a remarkable confirmation in the first observation of its optical spectrum by Heber et al. (1999) who found a line broadening of the order of $39 \mathrm{~km} \mathrm{~s}^{-1}$. But these data were obtained during an integration 
of $600 \mathrm{~s}$ on target (longer than the main $480 \mathrm{~s}$ period) and no measurements of radial velocity variations were available to discriminate between temperature, rotation and Doppler shift.

The possibility of detecting the radial velocity variations towards this star was first demonstrated by O'Toole et al. (2000, 2002) using a 2-m class telescope. Their analysis was based on the wavelength displacement of the Balmer lines and led them to detect seven oscillation velocities, most corresponding to previously-reported photometric modes. Adding the velocity amplitudes of the four dominant modes (around $2.1 \mathrm{mHz}$ and $2.7 \mathrm{mHz}$ ) lead then to an observable combined velocity amplitude of roughly $24 \mathrm{~km} \mathrm{~s}^{-1}$. However, despite the extended time-series obtained for this observation, a severe limitation on the precise amplitude measurement was set by the very low resolution available at the time ( $6 \AA$ at $4000 \AA)$. Additional observations with a 4-m class telescope (Anglo-Australian telescope) showed an apparent change in pulsation amplitude but, because of the short observing run, any attempt to use these observations for further asteroseismological work was compromised (Jeffery et al. 2003). To this day, it has been impossible to revisit the Kawaler's prediction on the rapid rotation of PG $1605+072$.

Continuing this pioneering work, O'Toole et al. (2003) presented the detection of line-index variations of Balmer lines. Because line-index variations are related to both $\delta T$ and $\delta \log g$, it was argued that such measurements may be used as an alternative to time-resolved spectroscopic data. Then, using a basic model of stellar pulsation, they proposed a temperature variation of about $490 \mathrm{~K}$, consistent with the previous observations of velocity amplitude.

In the following sections, after a brief presentation of the FUV spectrum and interstellar lines observed along the line of sight, we compute a composite lpv diagram of the star and compare our new results with the literature values.

\subsection{The FUV spectrum}

PG $1605+072$ is by far the brightest sdB star observed with FUSE and is thus the most amenable to spectroscopic study. An interstellar component is clearly identified along the line of sight within which small amounts of molecular hydrogen are detected: $\log N_{\mathrm{H} 2} \approx 18.7 \mathrm{~cm}^{-2}$ with a total $b$-value of $6.6 \mathrm{~km} \mathrm{~s}^{-1}$. This diffuse molecular component is observed through the first 4 rotational levels of the molecular hydrogen and is well described by a single excitation temperature of $130 \mathrm{~K}$. With such a low excitation temperature and an average velocity shift of $+23 \mathrm{~km} \mathrm{~s}^{-1}$ relative to the photospheric lines, it is unlikely that this diffuse molecular component is physically linked to PG $1605+072$. These numerous $\mathrm{H}_{2}$ absorption lines that plague the FUV spectrum make the analysis of the stellar lines difficult but, after careful inspection, we can identify a handful of useful photospheric lines: N III doublet at $100.60 \mathrm{~nm}, \mathrm{~S}$ III* at $101.56 \mathrm{~nm}$ and $101.58 \mathrm{~nm}, \mathrm{~S}$ IV at $106.27 \mathrm{~nm}$, and $\mathrm{S} \mathrm{IV}^{*}$ at $107.30 \mathrm{~nm}$ and $107.35 \mathrm{~nm}$ (see Table 2). Because the photospheric lines are highly structured and mildly saturated it is not possible to derive accurate estimates of the column density, temperature and b-value for each
Table 2. Identification of some photospheric lines in the spectrum of PG 1605+072.

\begin{tabular}{ccr}
\hline \hline $\begin{array}{c}\text { Species } \lambda \\
\mathrm{nm}\end{array}$ & $f_{\lambda}$ & $\begin{array}{r}\text { Equivalent Width } \\
\mathrm{m} \AA\end{array}$ \\
\hline N III100.60 & $3.76 \mathrm{e}-2$ & $105 \pm 10$ \\
S III*101.56 & $\ldots$ & $30 \pm 5$ \\
S III*101.58 & $\ldots$ & $30 \pm 5$ \\
S IV 106.27 & $5.8 \mathrm{e}-1$ & $45 \pm 6$ \\
S IV*107.30 & $5.2 \mathrm{e}-1$ & $120 \pm 10$ \\
S IV*107.35 & $5.7 \mathrm{e}-2$ & $30 \pm 5$ \\
\hline
\end{tabular}

ion. Other important information can however be gained from the analysis of the lpv diagram.

\subsection{The composite Ipv diagram}

To derive the lpv diagram towards PG 1605+072, the 4 strongest photospheric lines (Table 2.) are co-added and the alignment performed by cross-correlating over the numerous $\mathrm{H}_{2}$ absorption lines present around each stellar line. To optimize the signal-to-noise ratio, we divide the main pulsation period into 8 phase intervals of 60 seconds $(T=480 \mathrm{~s}$; $f=2.1 \mathrm{mHz}$ ). Figure 4 shows the resulting composite lpv diagram.

A remarkable feature of the derived lpv is its relative simplicity, at variance with the complexity usually exhibited by relatively high order $(l>2)$ non radial mode stars (Mantegazza et al. 2002). Although fine structures might be undetected at the FUSE resolution $\left(15 \mathrm{~km} \mathrm{~s}^{-1}\right)$ the actual $\mathrm{lpv}$ is not inconsistent with the presence of a dominant large amplitude radial mode. To allow for a more quantitative approach a simultaneous profile fitting of the highly ionized species was performed for each phase interval assuming that the photospheric lines are well approximated by a simple Voigt profile. The profile fittings showed that the central velocity of the stellar lines roughly follows a single sinusoid with $17 \mathrm{~km} \mathrm{~s}^{-1}$ amplitude which, at face value, translates into a radial amplitude of about $2600 \mathrm{~km} \mathrm{(1 \%}$ of the stellar radius). This result is an oversimplification since at least 4 nearby dominant velocity modes are present but it is consistent with velocity variations reported by O'Toole et al. (2002) using 2 m class telescopes and by Woolf et al. (2002) using $4 \mathrm{~m}$ class telescopes.

Using simultaneous information of photometric and velocity amplitudes, we are also able to derive in a straightforward manner the phase difference of the maximum flux amplitude relative to maximum radius amplitude and we find from a sinusoid fits (Fig. 5) $\phi=180 \pm 5$ degrees. This computation is remarkably consistent with the expected phase opposition which is the signature of an adiabatic process.

In a previous spectroscopic investigation of this star, Heber et al. (1999) reported a total $b$-value of the order of $39 \mathrm{~km} \mathrm{~s}^{-1}$ but, as Fig. 4 shows, those studies performed at low speed are biased by the rapid Doppler shift (in a $600 \mathrm{~s}$ integration on target, we expect to find a total $b$-value of the order of two times $17 \mathrm{~km} \mathrm{~s}^{-1}$, i.e. $34 \mathrm{~km} \mathrm{~s}^{-1}$, close to the reported value of $39 \mathrm{~km} \mathrm{~s}^{-1}$ ). Indeed our systematic profile fitting of the 


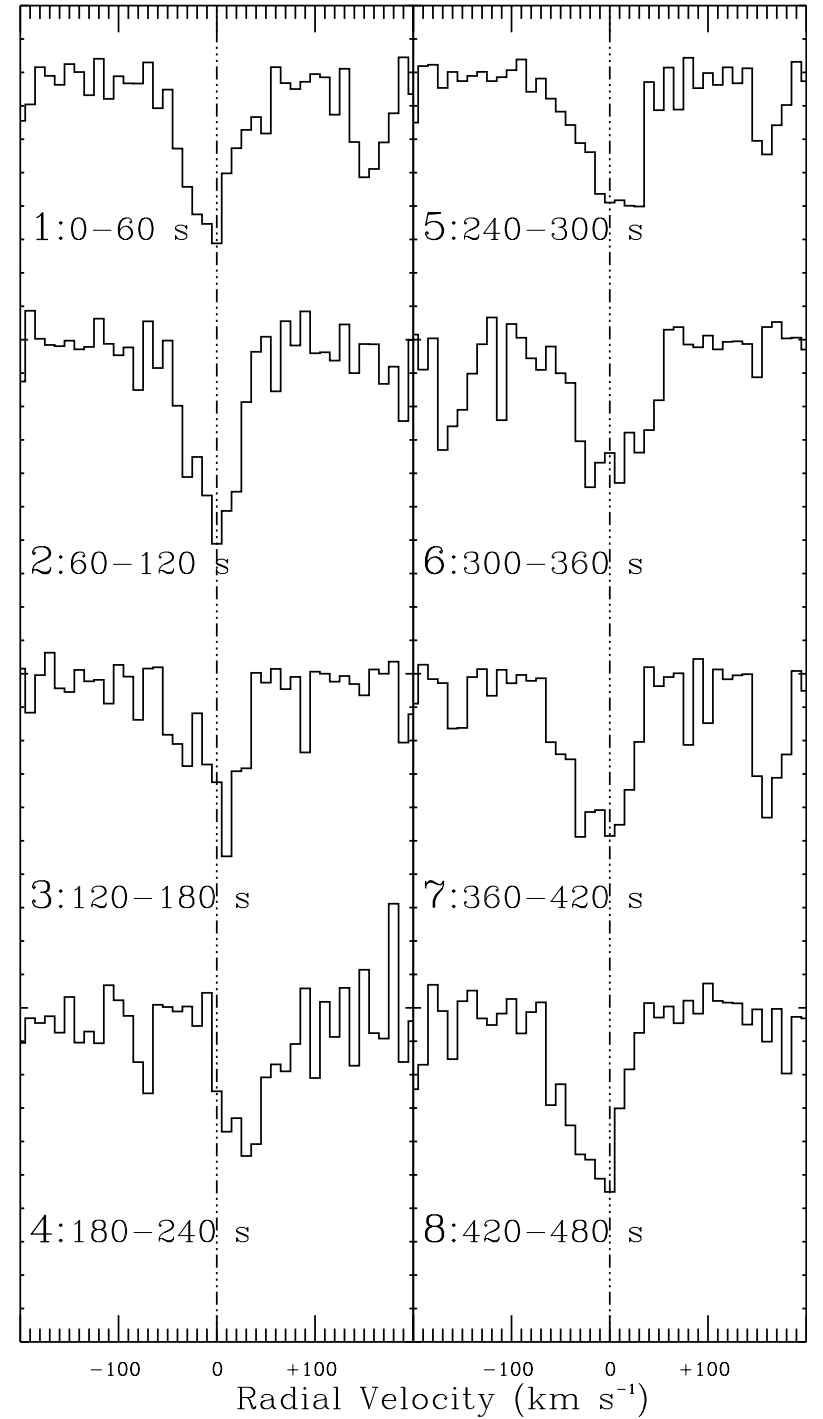

Fig. 4. Line profile variations observed at the surface of PG $1605+072$. Each panel represents a $60 \mathrm{~s}$ integration bin and each plotted profile, binned by $10 \mathrm{~km} \mathrm{~s}^{-1}$, is an average profile obtained from the coaddition of 4 strong photospheric lines: N II doublet at $100.60 \mathrm{~nm}, \mathrm{~S} \mathrm{III*}$ at $101.56 \mathrm{~nm}, \mathrm{~S} \mathrm{IV}$ at $106.27 \mathrm{~nm}$, and S IV* at $107.30 \mathrm{~nm}$. The central dotted line, around which the profiles move, shows the average zero velocity of photospheric species.

photospheric lines (see phases 2 and 3 in Fig. 4) leads to a much smaller $b$-value of $21 \pm 9 \mathrm{~km} \mathrm{~s}^{-1}$ (2- $\sigma$ error bars).

From the theoretical point of view, the low value we derive for the total $b$-value does not fit easily into the current pulsation models for PG 1605+072 (Kawaler et al. 1999; Kilkenny et al. 1999). In this model, low-order non-radial pulsations are the signature of trapped modes associated with an equatorial velocity of $130 \mathrm{~km} \mathrm{~s}^{-1}$. Such a high velocity is inconsistent with our derived b-value unless we assume a rather special geometry, with the star being observed almost pole-on $(i \approx 10 \mathrm{deg})$. We note also that such a large rotational velocity would be unique among sdB stars. An alternative model has recently been put forward based on the tentative detection of closely spaced frequencies within the $2.7 \mathrm{mHz}$ peak (Woolf et al. 2002), possibly due to rotational splitting of a multiplet. Then assuming

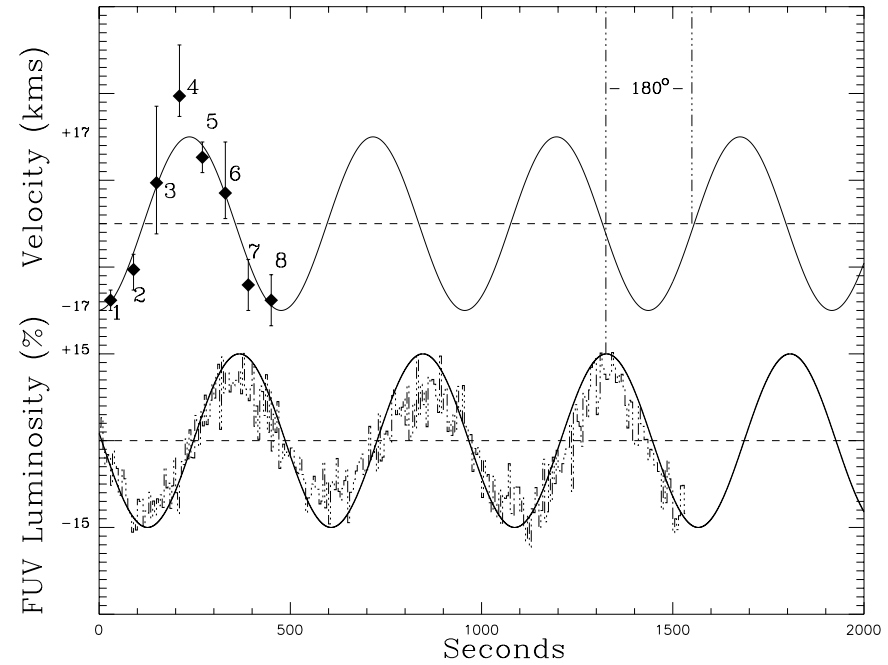

Fig. 5. Radial velocity and luminosity variation curves derived from the PG $1605+072$ observation. The radial velocities are derived from profile fitting of the lpv diagram (plotted error bars are 1- $\sigma$ ) while the FUV photometric data shown above are directly obtained from our special calibration procedure (see Sect. 3). The two vertical dotted lines help to compare the maximum luminosity and minimum radius position in phase. We note that both maxima are in phase opposition as expected from adiabatic pulsations.

that the frequency splitting between modes with successive $\mathrm{m}$ values is $\delta v \approx v_{\text {rot }} /[l(l+1)] \approx 0.01 \mathrm{mHz}$, where $v_{\text {rot }}$ is the rotational frequency, and assuming $R=0.28 R_{\odot}$ (Woolf et al. 2002), we note that a $l=1$ mode split into $m=0, \pm 1$ leads to $V_{\text {rot }}=21-23 \mathrm{~km} \mathrm{~s}^{-1}$. Indeed, such a rotational velocity seems roughly consistent with our observation but better quality data are needed for an unambiguous mode identification.

\section{Future prospects for space-based asteroseismology with FUSE}

The new results reported in this work have been made possible thanks to the unexploited photometric capabilities of the FUSE satellite. FUSE is equipped with two microchannel plate detectors ( 90000 by 200 resolution elements each) and can handle a total counting rate of the order of 32000 events per second with a quantum efficiency of $15-30 \%$ in the $90-120 \mathrm{~nm}$ range (Sahnow et al. 2000). In time-tagged mode operation it is possible to achieve a photometric signal-to-noise ratio of about 1000 in $30 \mathrm{~s}$.

FUSE is not the only instrument that can be diverted to provide photometric data and a few prior attempts to detect pulsations from space have been reported with HST towards white dwarf stars as early as 1995 (see Robinson et al. 1995). Alas, given the present status of servicing missions to $H S T$, FUSE will soon be the only instrument of its kind. In the coming years, more datasets will become available thanks to dedicated missions such as COROT (Baglin et al. 2001) and MOST (Walker et al. 2003), but will these missions totally eclipse the potential of the FUSE satellite for asteroseismology or will FUSE remain useful? 
Table 3. FUSE, MOST and COROT photometric performances.

\begin{tabular}{lrccc}
\hline \hline & $\begin{array}{c}\text { FUSE } \\
\text { Archive }^{*}\end{array}$ & $\begin{array}{c}\text { FUSE } \\
\text { Legacy }^{* *}\end{array}$ & MOST & COROT \\
& $9000 \mathrm{~s}$ & 4 days & 32 days & 150 days \\
Exposure time & $110 \mu \mathrm{Hz}$ & $2.5 \mu \mathrm{Hz}$ & $0.36 \mu \mathrm{Hz}$ & $0.08 \mu \mathrm{Hz}$ \\
Frequency resolution & $1 \mathrm{~Hz}$ & $1 \mathrm{~Hz}$ & $83 \mathrm{mHz}$ & $31 \mathrm{mHz}$ \\
Maximum sampling rate & & & & \\
\hline 2- $\sigma$ detection (ppm) & 5000 & 800 & $\ldots$ & $\ldots$ \\
WD, $M v=15$ & 700 & 100 & 4600 & 4.8 \\
O9 star, $M v=9$ & $\ldots$ & $\ldots$ & 290 & 1.2 \\
G2 star, $M v=6$ & & & & \\
\hline
\end{tabular}

* An exposure time of 9000 is typical of many archived FUSE spectra.

** An exposure of 4 continuous days assuming a duty cycle of $50 \%$ leads to $180000 \mathrm{~s}$ of total exposure time. Such observation program has not been achieved yet with FUSE towards a pulsating stars but may be planned as part of a FUSE Legacy program.

Table 3 shows a simulation of performances of FUSE, MOST and COROT for three typical targets: a 15th mag white dwarf $(T=40000 \mathrm{~K})$, a 9th mag O9 star and 6th magnitude solar type star $(\mathrm{G} 2)$. The comparison is based on routine operations: from a $9000 \mathrm{~s}$ continuous exposure with FUSE to a 6 months observation with COROT.

The reported 2- $\sigma$ detection in the Fourier space has been extrapolated from prelaunch performances for COROT (Baglin et al. 2001) and the MOST in-flight performances we adopt are extrapolated from the recent photometric results achieved towards Procyon (Matthews et al. 2004) which seem rather modest compared to prelaunch estimates; hence, a 2- $\sigma$ detection of the order of 6 ppm (part per million) is obtained above $2 \mathrm{mHz}$ for a $V=0.38$ star (Procyon).

To compute the FUSE detection capabilities we estimated the total fluxes for each target following the reliable estimates available on-line at the FUSE web site. Then adding white noise consistent with the expected signal-to-noise ratio we buried a $10 \mathrm{mHz}$ mode of constant amplitude in the FUV timeseries. In order to obtain realistic time-series we divided each FUSE observation into chunks of 9000 s each separated by gaps of $9000 \mathrm{~s}$. We then report the detectability of the buried mode in the Fourier space.

As Table 3 shows, the estimated performances of the COROT photometer are several orders of magnitude better than any other instrument for stars brighter than the 9th mag. But FUSE is indeed better suited for faint star pulsation studies: FUSE will be the only instrument making faint $(M v>13)$, short period (less than $60 \mathrm{~s}$ ) pulsators amenable to study. Its design and mission planning have three other unique advantages: 1) it can routinely perform several snapshot observations of 4000 seconds in a row with a much higher sensitivity than any ground-based facility; 2) it offers the fastest sampling rate ever achieved in space (up to $1 \mathrm{~Hz}$ ); and 3) it can provide high-speed time-resolved spectroscopic data in the FUV domain where many photospheric lines occur. To our knowledge these latter performances will have no equivalent among space telescopes for the next decade.

As of today, about 1500 different stars have been observed with the FUSE satellite in time-tagged mode among which dozens of sdB stars (see http:// achive.stsci.edu/fuse/). Each of these datasets can be converted into FUV photometric time-series following the calibration method described in this paper. Many of these datasets could provide high-speed time-resolved spectroscopic data as well. Given the large sample of targeted stars available in the archive, the asteroseismological potential of the FUSE satellite may well extend far beyond sdB investigations.

\section{Summary}

1. Important progress has been made thanks to the many FUV spectra of dwarf stars obtained by FUSE (Fontaine \& Chayer 2004). In this paper, we present an original way of handling FUSE data that allows one to fully exploit its highspeed photometric capabilities. We report on the detection of two dominant modes towards PG 1219+534 (at $6.9 \mathrm{mHz}$ and $7.8 \mathrm{mHz}$ ) and towards PG 1605+072 (at $2.1 \mathrm{mHz}$ and $2.7 \mathrm{mHz}$ ) and we confirm the existence of a single dominant mode towards PG $1613+426$ at $7.048 \pm 0.3 \mathrm{mHz}$.

2. We further report on the Doppler shift of the photospheric lines at the surface of PG $1605+072$ with $17 \mathrm{~km} \mathrm{~s}^{-1}$ amplitude. The time-resolved spectroscopy of this star allows us to derive the projected broadening of the photospheric lines with unprecedented accuracy: $v \sin i=21 \pm 9 \mathrm{~km} \mathrm{~s}^{-1}$ (2- $\sigma$ error bar). We note that this result is only marginally consistent with the current theoretical expectations that the rapid rotation of $P G 1605+072$ is the source of its pulsation pattern. Thanks to these new data we are also able for the very first time to observe the phase opposition between the maximum radius and the maximum velocity as expected if adiabatic pulsations are taking place in the envelope of PG $1605+072$.

3. Finally, we demonstrate that FUSE is particularly well suited for asteroseismological investigation of dwarf stars in general. Comparing the actual FUSE photometric performances with MOST and COROT, we conclude that FUSE is a unique instrument for the study of faint $(M v>10)$, hot ( $T>20000 \mathrm{~K})$ pulsating objects and should be considered an alternative to the COROT mission in that observation domain.

Acknowledgements. We are very grateful to James Caplinger and Bryce Roberts from the FUSE staff for valuable discussions on the FUSE detectors and mission planning. Kuassivi is especially grateful to Farid Abdelfettah, Sylvie André, Jean-Michel Arslanian and Olivier Rullière from the AZimov association for enlightening comments about the general science reported in this paper. The work at the Institut d'Astrophysique de Paris was supported by CNES/CNRS/UPMC. The work at the Osservatorio Astrofisico di Catania was supported by the Italian Ministero dell'Istruzione, Università e Ricerca. 


\section{References}

Baglin, A., Auvergne, M., Catala, C., Michel, E., \& COROT Team 2001, in SOHO/GONG Workshop

Bonanno, A., Catalano, S., Frasca, A., Mignemi, G., \& Paternò, L. 2003, A\&A, 398, 283

Brown, T. M., Bowers, C. W., Kimble, R. A., Sweigart, A. V., \& Ferguson, H. C. 2000, ApJ, 532, 308

Charpinet, S., Fontaine, G., Brassard, P., \& Dorman, B. 1996, ApJ, 471,103

Fontaine, G., \& Chayer, P. 2004 [arXiv: astro-ph/0411091]

Heber, U., \& Kudritzki, R. P., 1986, A\&A, 169, 244

Heber, U., Reid, I. N., \& Werner, K. 1999, A\&A, 348, L25

Jeffery, C. S. 2004, Ap\&SS, 291, 403

Kawaler, S. D. 1999, 11th European Workshop on White Dwarfs (ASP), ASP Conf. Ser., 169, 158

Kepler, S. O., Robinson, E. L., Koester, D., et al. 2000, ApJ, 539, 379

Kilkenny, D., Koen, C., O'Donoghue, D., \& Stobie, R. S. 1997, MNRAS, 285, 640

Kilkenny, D., Koen, C., O’Donoghue, D., et al. 1999, MNRAS, 303, 525
Koen, C., O’Donoghue, D., Pollacco, D. L., \& Charpinet, S. 1999, MNRAS, 305, 28

Kuassivi, Bonanno, A., Ferlet, R., Roberts, B., \& Caplinger, J. 2004 [arXiv: astro-ph/0411371]

Mantegazza, L., \& Poretti, E. 2002, A\&A, 396, 911

Matthews, J. M., Kusching, R., Guenther, D. B., et al. 2004, Nature, 430

Moos, H. W., Cash, W. C., Cowie, L. L., et al. 2000, ApJ, 538, L1

O'Toole, S. J., Bedding, T. R., Kjeldsen, H., et al. 2000, ApJ, 537 , L53

O’Toole, S. J., Bedding, T. R., Kjeldsen, H., Dall, T. H., \& Stello, D. 2002, MNRAS, 334, 471

O’Toole, S. J., Jørgensen, M. S., Kjeldsen, H., Bedding, T. R, Dall, T. H., \& Heber, U. 2003, MNRAS, 340, 856

Robinson, E. L., Mailloux, T. M., Zhang, E., et al. 1995, ApJ, 438, 908

Sahnow, D. J., \& the FUSE team 2000, ApJ, 538, L7

Walker, G., Matthews, J., Kuschnig, R., et al. 2003, PASP, 115, 1023

Woolf, V. M., Jeffery, C. S., \& Pollacco, D. L. 2002, MNRAS, 329, 497 